This item was submitted to Loughborough's Research Repository by the author.

Items in Figshare are protected by copyright, with all rights reserved, unless otherwise indicated.

\title{
An investigation into the wake structure of square back vehicles and the effect of structure modification on resultant vehicle forces
}

PLEASE CITE THE PUBLISHED VERSION

http://dx.doi.org/10.4271/2011-37-0015

PUBLISHER

(C) SAE International

VERSION

VoR (Version of Record)

LICENCE

CC BY-NC-ND 4.0

\section{REPOSITORY RECORD}

Littlewood, Rob, Martin A. Passmore, and Daniel Wood. 2012. "An Investigation into the Wake Structure of Square Back Vehicles and the Effect of Structure Modification on Resultant Vehicle Forces". figshare. https://hdl.handle.net/2134/9850. 
This item was submitted to Loughborough's Institutional Repository (https://dspace.lboro.ac.uk/) by the author and is made available under the following Creative Commons Licence conditions.

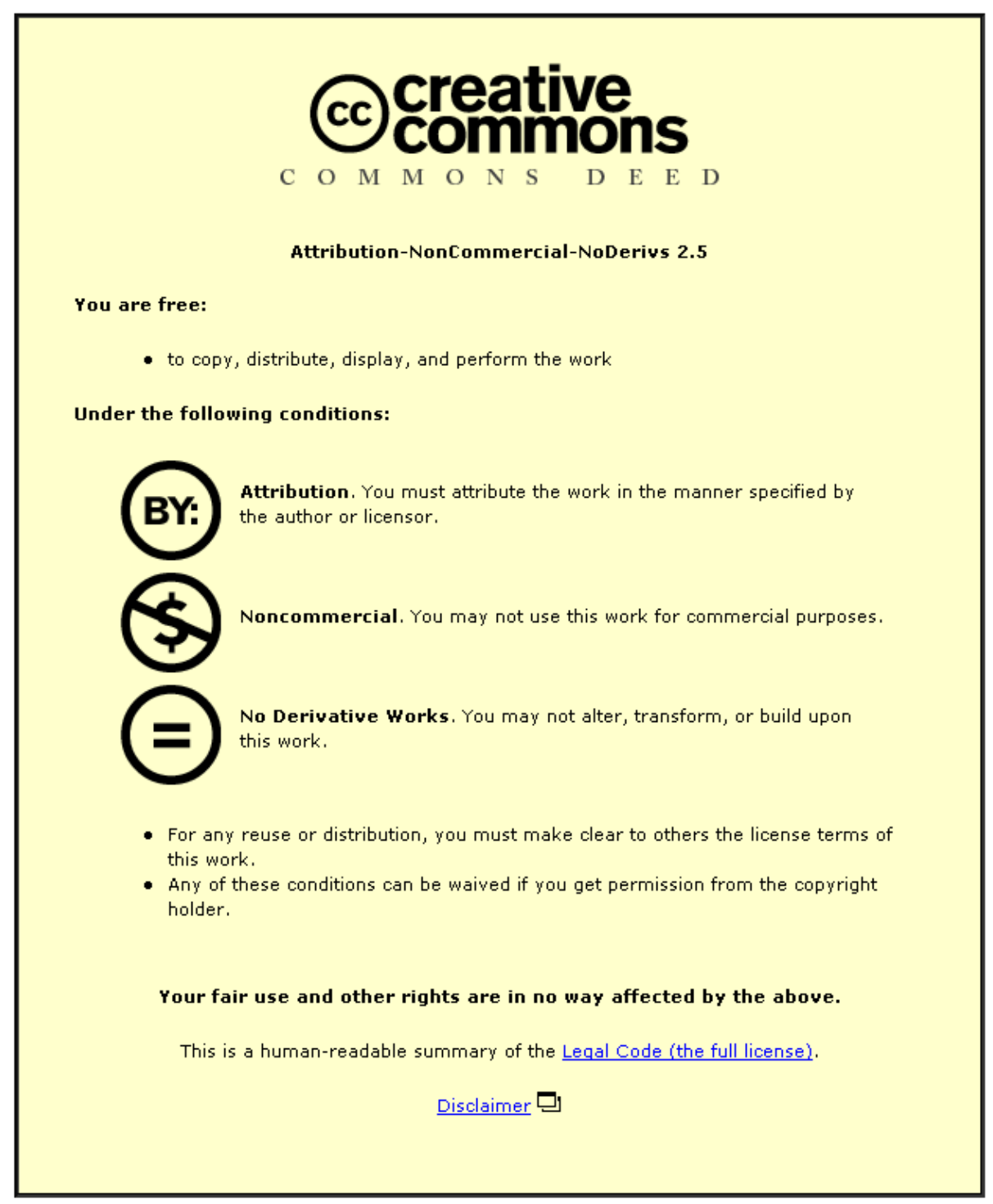

For the full text of this licence, please go to: http://creativecommons.org/licenses/by-nc-nd/2.5/ 


\section{An Investigation into the Wake Structure of Square Back Vehicles and the Effect of Structure Modification on Resultant Vehicle Forces}

Rob Littlewood

Loughborough University

Martin Passmore

Loughborough Univ

Daniel Wood

Loughborough University

Copyright $(C 2011$ SAE International

\section{ABSTRACT}

A large contribution to the aerodynamic drag of a vehicle (30\% 1 ) or more depending on vehicle shape) arises from the low base pressure in the wake region, especially on squareback configurations. A degree of base pressure recovery can be achieved through careful shape optimization, but the flow structures and mechanisms within the wake that cause these base pressure changes are not well understood. A more complete understanding of these mechanisms may provide opportunities for further drag reductions from both passive shape changes and in the future through the use of active flow control technologies.

In this work surprisingly large changes in drag and lift coefficients of a square-back style vehicle have been measured as a result of physically small passive modifications. Tests were performed at quarter scale using a simplified vehicle model (Windsor Model) and at full scale using an MPV. The full scale vehicle was tested with and without a flat floor. During both tests the vehicle base region was fitted with a series of low profile, horizontal slats to disrupt any steady wake structures acting close to the vehicle base surface. Force balance, static pressure and PIV measurements have been used to investigate the flow structures in the vehicle wake.
This paper summarizes the results and relates the global drag and lift changes that arise from the horizontal slats to the base pressure from both model and full scale and to the PIV measurements from the model tests.

\section{INTRODUCTION}

It is clear that the reduction of $\mathrm{CO}_{2}$ emissions from road vehicles is currently, and will continue to be, a significant area of research for the automotive industry. The effect on $\mathrm{CO}_{2}$ emissions from fossil fuelled vehicles as a result of reduced aerodynamic drag forces is dependent on what drive cycle is used in the calculation(2), but can be significant. The effect of aerodynamic drag also becomes important when considering vehicle range requirements for low energy density power sources such as batteries and fuel cells.

It is worthy of note that whilst aerodynamic drag coefficients $\left(C_{d}\right)$ have fallen significantly over the past thirty years, vehicle frontal areas have seen an increase $(\underline{3})$. This inverse tendency illustrates the difficult tradeoffs faced by vehicle manufacturers when considering consumer requirements and buying trends, but it is apparent that vehicle package and styling are still dominant factors when creating vehicle body shapes. 
For this reason the vehicle aerodynamicist must use ever more discreet means of achieving drag reductions such as air spoilers, wheel deflectors $(\underline{4})$, underbody smoothing $(\underline{5})$ and sealing of under bonnet gaps $(5)$. In recent years active flow control techniques have been of research interest due to their potential ability to achieve flow modification and drag reductions without the need for physical shape changes, however they have yet to be proven in a real world vehicle application. Previous experiments using the Windsor reference model and steady blowing $(\underline{6})$ as a means of flow control have found large reductions in drag forces, that arise through modification of the wake structure to create an increase in base pressure. Littlewood $(\underline{6})$ reported that the mechanisms that appear to be important in these changes might also be effected through passive modifications. Therefore the current work uses passive techniques with the aim of modifying the wake structure and are used as a means of understanding how flow mechanisms and structures within the wake of a squareback vehicle can influence drag forces.

The majority of the experimentation has been conducted at $1 / 4$ model scale, however the opportunity arose to conduct limited tests on a full scale vehicle, so these have been reported here. Within the limited time available to test at full scale only a small number of configurations could be tested, however the results verify that the effects seen are not exclusive to model scale testing. Although the findings are a means of understanding wake structure modification, the small passive modifications made could have implications or an application within a passive optimisation of current passenger or haulage vehicles.

\section{BACKGROUND}

In previous investigations $(\underline{7})$ the author has linked the modification of wake size via a small passive optimisation to an increase in base pressure and as such a reduction in vehicle drag. This is illustrated by Figure 1a) and $\underline{1 b}$ ) which show PIV images on streamwise centreline planes in the near wake of the $1 / 4$ scale square back Windsor model. Figure $1 \mathrm{~b}$ ) illustrates the wake size reduction when a 12 degree high aspect ratio slant is applied to the roof trailing edge. This small passive modification results in a $\mathrm{C}_{\mathrm{d}}$ reduction of 12 counts. a)

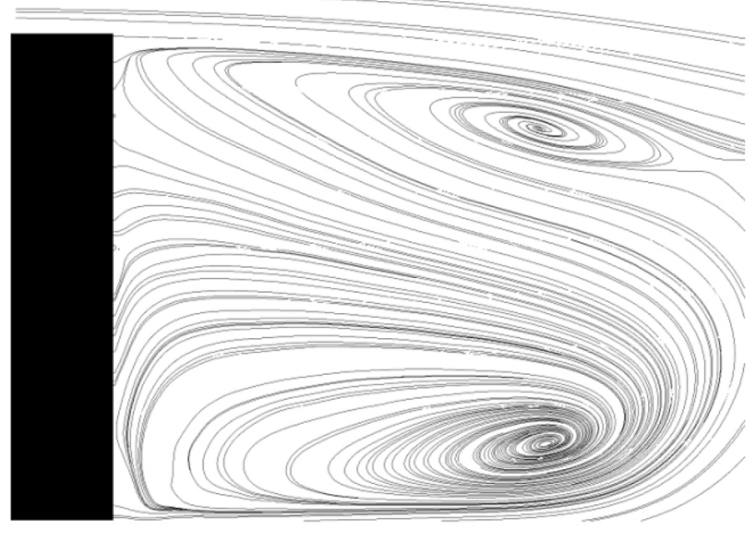

0.7

0.6

0.5

0.4
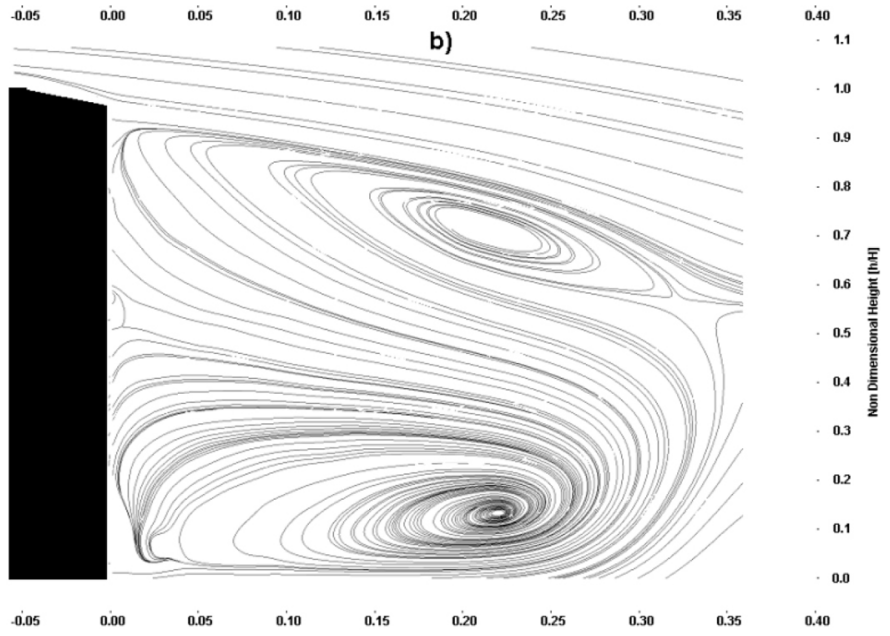

Figure 1. a) Time averaged PIV streamlines of squareback wake structure; b) Time averaged PIV streamlines of squareback wake structure incorporating a $12^{\circ}$ roof trailing edge chamfer

Previous investigations have tried to link the size of the wake of bluff bodies to the pressure seen on the body base $(\underline{8})(\underline{9})$. Some studies argue larger wakes lead to increased base pressure while others claim the opposite. In the case of the work reported in Littlewood et al $(\underline{7})$ the wake size is inferred from PIV data from a single centreline plane, and it is concluded that the reduction in overall wake size is linked to higher base pressures. The conclusion is supported by the detailed base pressure data.

Later experiments using constant blowing applied at the rear roof trailing edge $(\underline{6})$ of the same model generated $C_{d}$ reductions of up to 35 counts, or approximately $10 \%$. However when PIV images were taken of the blown and unblown case the wake size did not decrease as much as might have been expected from the passive optimisation studies for such a large $\mathrm{C}_{\mathrm{d}}$ change. The baseline wake structures differ slightly between Figure 1 and Figure 2 
because the model underbody conditions were modified between the tests.

a)

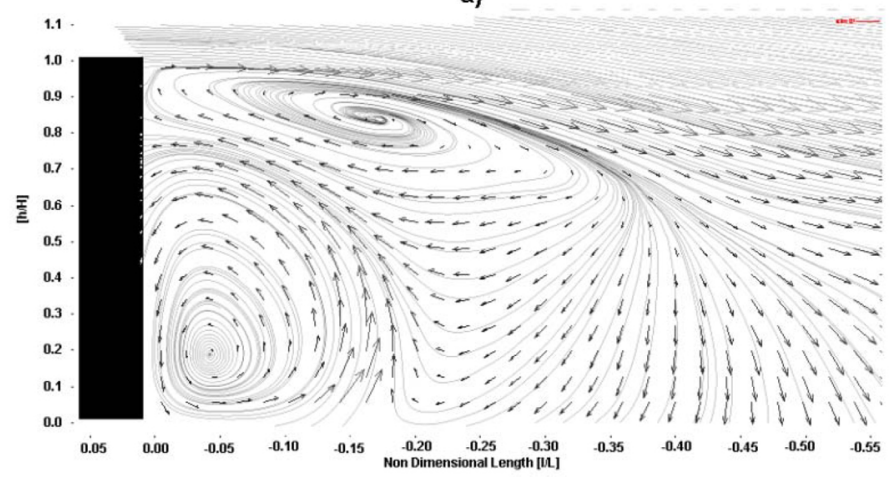

b)

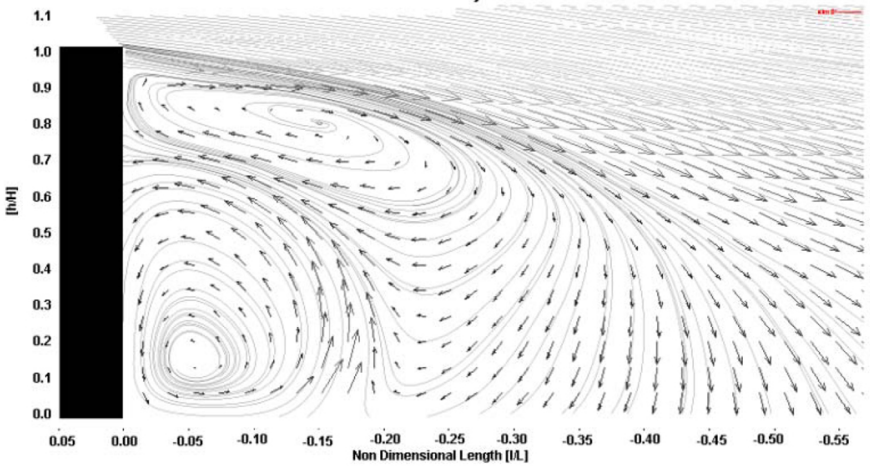

Figure 2. a)Time averaged PIV of baseline case squareback wake structure b)Time averaged PIV of constant blowing case, squareback wake structure

Describing the entire wake structure in line with the 'cavity model' outlined by Rhosko( ${ }^{(8)}$ it would seem that the global 'in wake' or 'in cavity' pressure can be influenced by overall wake/cavity size. However if this is the case, for the configurations shown in Figure 2a) and $\underline{2 b}$ ) it might be reasonable to expect a larger change in wake size than is observed for such significant $C_{d}$ changes. From this analysis it is apparent that the pressures seen on the base surface of the model must be influenced by more than just wake size alone. It can be seen from Figure 2 that the time averaged vortex structures close to the vehicle base impart a large flow velocity close to the base surface which in turn creates a suction component. It was hypothesized that a means of interrupting or slowing the surface flow velocity on the base may lead to an increase in base pressure in this region of suction.

The current work uses small passive modifications to the base of the model in an attempt to disrupt the flow velocity close to the model base. The objective is to show that disrupting the wall velocities can have an influence on wake structure and hence, base pressure. Additionally the study aims to demonstrate a new passive method for influencing base pressures other than altering the wake size alone.

\section{EXPERIMENTAL SETUP AND DATA ACQUISITION}

\section{MODEL SCALE TESTING}

The Windsor model $(\underline{10})$, used in this work is shown in Figure $\underline{3}$. All the leading edges are well rounded to avoid separation with radii of $0.05 \mathrm{~m}$, with the exception of the roof leading edge which has a radius of $0.20 \mathrm{~m}$. The longitudinal and rear edges are sharp. A NACA0021 wing profile with $150 \mathrm{~mm}$ chord protrudes from the centre of the model floor, to below the wind tunnel floor. This wing is fitted as shielding for tubing and cabling that may need to be routed inside the model. The standard configuration of the base surface is flat and perpendicular to the onset flow direction. The model was fitted with small $1 \mathrm{~mm}$ thick, $8 \mathrm{~mm}$ deep slats on the lower half of its base surface, as shown in detail A of Figure 3. The first slat added was positioned $35 \mathrm{~mm}$ above the model floor and additional slats were spaced $35 \mathrm{~mm}$ apart. The configurations tested are described in Table 1.
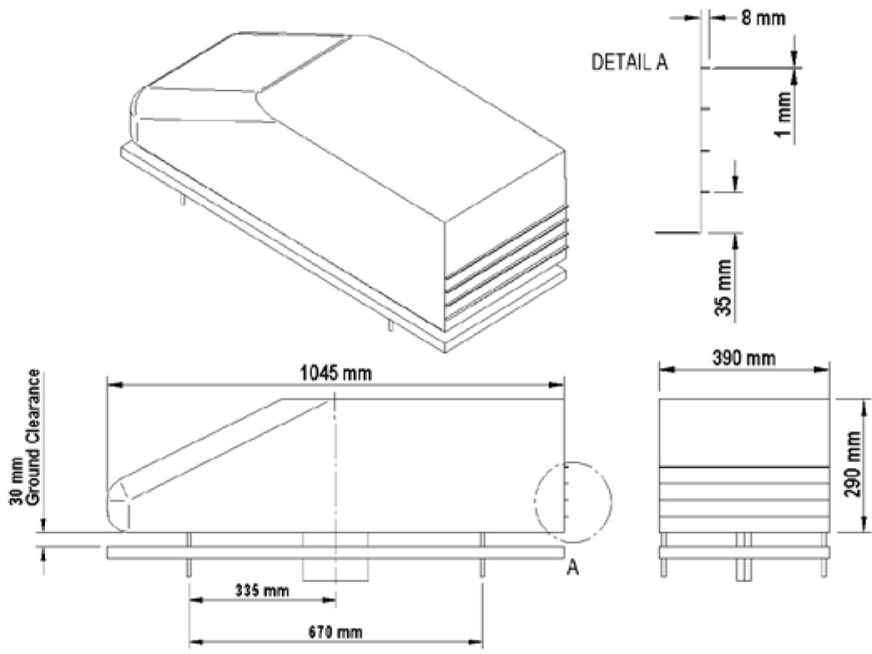

Figure 3. Windsor model illustrating the position of slats

Table 1. Scale model configurations

\begin{tabular}{|c|c|}
\hline $\begin{array}{c}\text { Configuration } \\
\text { Number }\end{array}$ & Description \\
\hline 0 & $\begin{array}{c}\text { Baseline configuration no slats } \\
\text { added to model }\end{array}$ \\
\hline 1 & $\begin{array}{c}\text { 3 Slats added to the base surface of } \\
\text { the model spaced evenly 35mm } \\
\text { apart from the floor of the model }\end{array}$ \\
\hline 2 & $\begin{array}{c}\text { 4 Slats added to the base surface of } \\
\text { the model spaced evenly 35mm } \\
\text { apart from the floor of the model }\end{array}$ \\
\hline
\end{tabular}


All scale tests were performed in a closed working section, open circuit wind tunnel with a fixed floor. The working section of the tunnel is $1.92 \mathrm{~m} \times 1.32 \mathrm{~m}$ which gives a blockage of $4.4 \%$ for this model. Force measurements were made using a six component under-floor balance that was coupled to the model using four $8 \mathrm{~mm}$ diameter pins. The standard ride height chosen for the tests was $30 \mathrm{~mm}$ giving a nondimensional ride height of $10.3 \%$ based on the model height. A Reynolds sweep was performed and established that the model was insensitive to length based Reynolds number variations above approximately 1.4 million. The subsequent tests were performed with freestream velocities of 30,35 and $40 \mathrm{~m} / \mathrm{s}$, corresponding to Reynolds numbers of 2.15 million, 2.51 million and 2.87 million respectively. All coefficients have been based on model frontal area and values have been corrected for blockage using the area ratio method as expressed in Equation (1 where $\mathrm{E}$ is the ratio of model frontal area to working section cross sectional area.

$$
C_{\text {Fcorrected }}=\frac{C_{\text {Fmeasured }}}{(1+2 E)}
$$

The model is equipped with 111 pressure tappings on the base of the model and are connected via flexible tube to two 64 channel pressure scanners mounted inside the model. Data was acquired from the scanners via CAN technology which required two ethernet cables to be routed through the wind tunnel floor and through the model floor at its centre-line. The static accuracy of the scanners range between \pm 0.06 to \pm 0.1 as a percentage of full scale depending on operating conditions. The full scale of the scanners was used in these tests to provide $\pm 232 \mathrm{mmH}_{2} \mathrm{O}$ and all pressure readings were corrected for blockage using Equation (2.

$$
C_{\text {Pcorrected }}=\frac{C_{P \text { measured }}+2 E}{(1+2 E)}
$$

\section{FULL SCALE VEHICLE TESTING}

The test vehicle was a commercially available square back MPV and experiments were conducted in the MIRA full scale test facility which is a fixed floor, closed working section, open return wind tunnel. The vehicle wheels were not rotating during the tests. The working section of the tunnel is $7.94 \mathrm{~m}$ wide and $4.42 \mathrm{~m}$ high, giving a blockage ratio of $7.6 \%$, using the manufacturer's quoted frontal area of $2.65 \mathrm{~m}^{2}$. Tests were performed at freestream velocities of 22.4, 26.8 and $31.3 \mathrm{~m} / \mathrm{s}(50,60$ and $70 \mathrm{mph})$. Force measurements were made using a six component under-floor balance and corrected for blockage using the standard MIRA blockage correction method. 30 surface pressure 'spades' were positioned across one half of the vehicle base and were connected via flexible tubing to a single 64 channel pressure scanner mounted inside the vehicle. The scanner specification was identical to that used in the model scale testing and measurements were non- dimensionalised using a pitot static tube located upstream of the vehicle. The pressure measurements were corrected using Equation (2.

The configurations tested at full scale consisted of adding up to six $30 \mathrm{~mm}$ deep slats across the width of the base as shown in Figure 4. The configurations tested are described in Table $\underline{2}$.

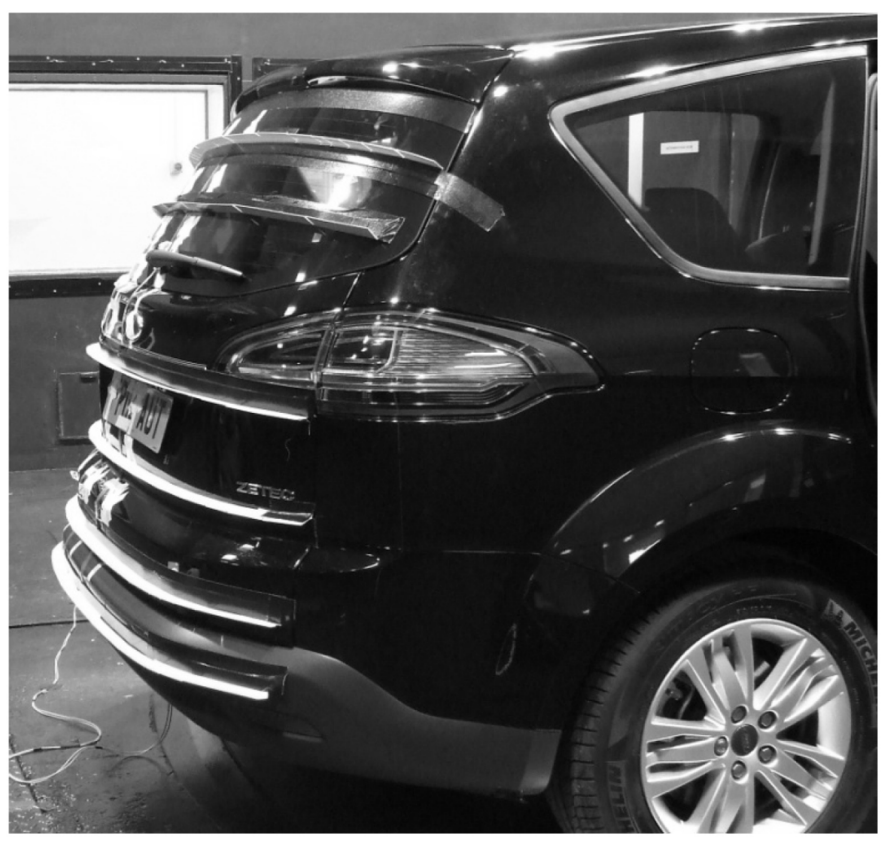

Figure 4. Full scale test vehicle shown in

\begin{tabular}{|c|c|}
\hline $\begin{array}{l}\text { Configuration } \\
\text { Number }\end{array}$ & Description \\
\hline 0 & $\begin{array}{l}\text { No slats fitted baseline vehicle } \\
\text { configuration }\end{array}$ \\
\hline 1 & $\begin{array}{c}6 \text { Slats fitted to the base surface } \\
\text { of the vehicle as shown in Figure } \\
4\end{array}$ \\
\hline 2 & $\begin{array}{l}5 \text { slats fitted to the base as per } \\
\text { configuration } 1 \text { but with the } \\
\text { upper slat removed }\end{array}$ \\
\hline 3 & $\begin{array}{l}4 \text { slats fitted to the base as per } \\
\text { configuration } 1 \text { but with the } \\
\text { upper slat removed }\end{array}$ \\
\hline 4 & $\begin{array}{l}3 \text { slats fitted to the base as per } \\
\text { configuration } 3 \text { but with the } \\
\text { upper slat removed }\end{array}$ \\
\hline 5 & $\begin{array}{l}2 \text { slats fitted to the base as per } \\
\text { configuration } 4 \text { but with the } \\
\text { upper slat removed }\end{array}$ \\
\hline 6 & $\begin{array}{l}1 \text { slat fitted to the base as per } \\
\text { configuration } 5 \text { but with the } \\
\text { upper slat removed }\end{array}$ \\
\hline
\end{tabular}

Table 2. Full scale vehicle configurations 


\section{EXPERIMENTAL RESULTS}

\section{MODEL SCALE}

The balance measured change in $C_{d}$ relative to the baseline configuration for the scale model tests are shown in Figure 5. For clarity error bars have been omitted from the graphs, however the measured $C_{d}$ values are accurate to \pm 1 count and measured lift coefficients $\left(\mathrm{C}_{1}\right)$ values are accurate \pm 5 counts. Slats were only applied to the lower half of the base during the model scale experiments. These configurations were chosen after identifying that a strong and relatively stable vortical structure is present in close proximity to the base surface, as can be seen in Figure 1, Figure 2 and Littlewood (). Configuration numbers are as described in Table 1.

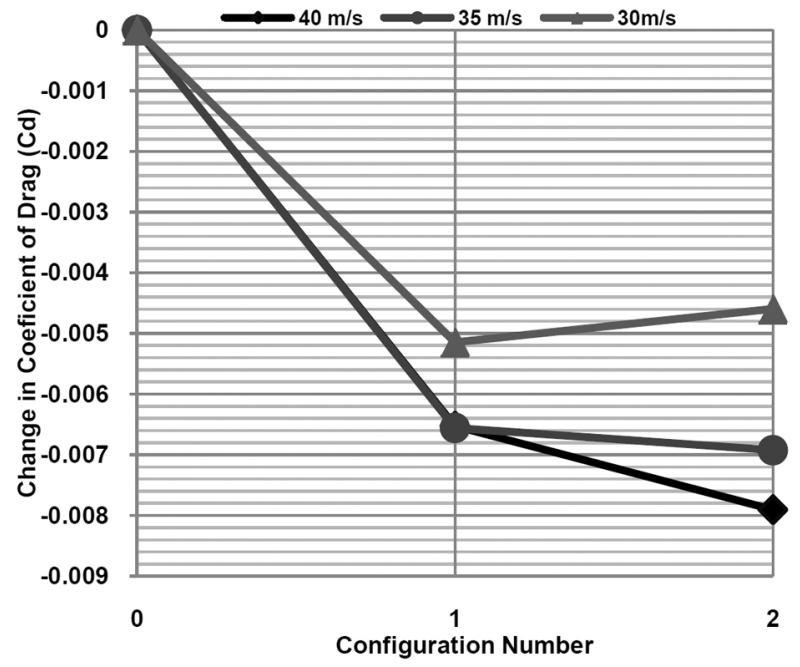

Figure 5. Change in Cd from baseline vs configuration number

Figure 5 shows that the addition of slats to the base of the model reduces $C_{d}$ by up to 8 counts for configuration 2 . This is a considerable reduction in overall $\mathrm{C}_{\mathrm{d}}$ for such a small shape change within the wake. Particularly as in previous studies $(11)$, shape changes within the wake intended to modify the structures of the wake have been considerably larger than those employed here. It is suggested that this drag change is the result of a modification to the structure of the wake, specifically the strong lower vortex structure seen in Figure 1 and Figure 2.

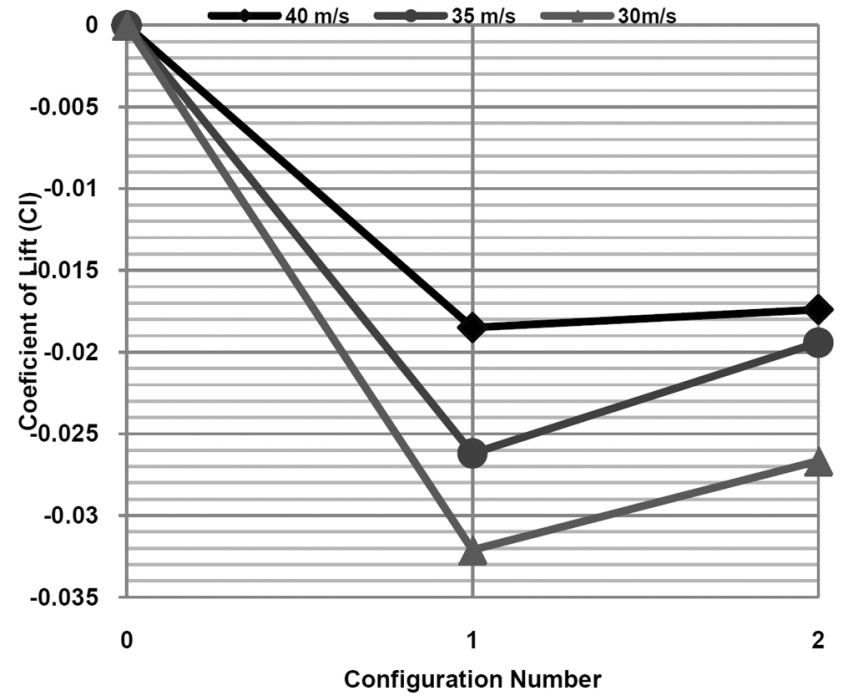

Figure 6. Change in Cl from baseline

Figure 6 shows the vehicle lift coefficient, and the effect of the configuration changes relative to the baseline configuration. An initial observation is that the $C_{1}$ values are much more sensitive to Reynolds number changes than the drag results, however they all follow a trend that with the addition of slats to the lower half of the base $\mathrm{C}_{1}$ is reduced. This is again a significant change in $\mathrm{C}_{1}$ for a relatively small shape change, and it supports the theory that disrupting the lower vortex in close proximity to the wall will cause a base pressure increase. It is possible that small modifications at the rear of the model can cause changes in the stagnation point at the front of the vehicle which could account for the changes in lift, however looking at the balance of front and rear $\mathrm{C}_{1}$ changes this effect is only a small percentage of the overall lift change. The rear lift coefficient $\left(\mathrm{C}_{\mathrm{lr}}\right)$ never contributes less than $80 \%$ to the total changes in $C_{1}$ recorded. This indicates that changes in lift due to movement of the front stagnation point are small, and the majority of the lift changes occur because of changes to the wake structure effecting the flow around the rear section of the vehicle.

Figure 7 shows a contour plot of base pressure coefficients (Cp) for the baseline configuration (no slats) and Figure 8 shows the $\mathrm{Cp}$ distribution when four slats are added (configuration 2). The plots are oriented such that a view is taken looking from directly behind the model where zero on the $\mathrm{x}$ axis corresponds to the model centreline and zero on the $\mathrm{z}$ axis corresponds to the bottom of the model. 


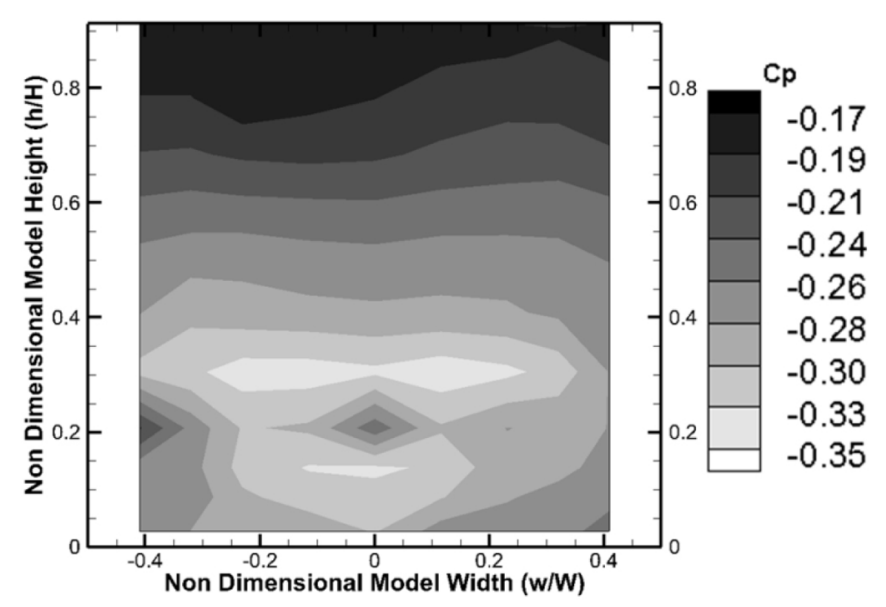

Figure 7. Cp distribution on model base with no slats fitted (freestream velocity $40 \mathrm{~m} / \mathrm{s}$ )

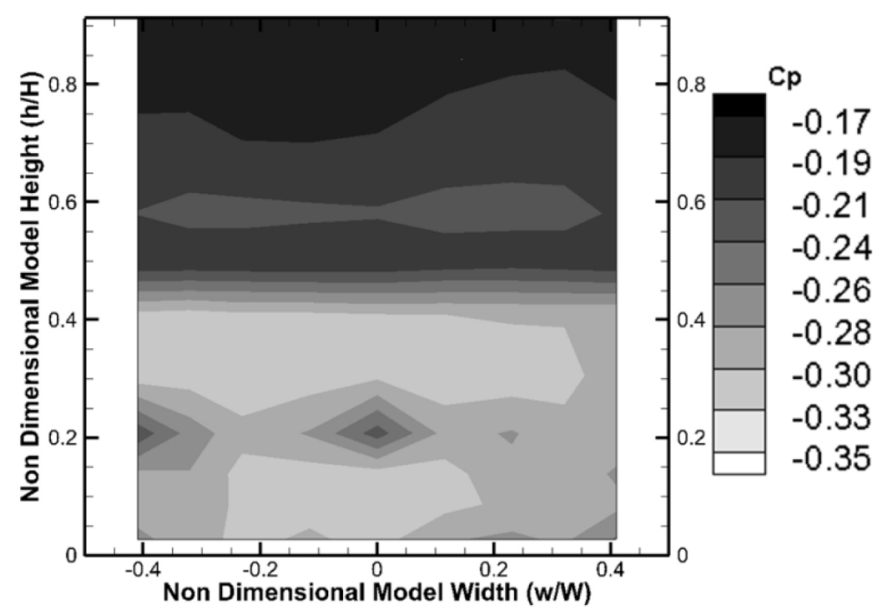

Figure 8. Cp distribution on model base with 4 slats fitted (freestream velovity $40 \mathrm{~m} / \mathrm{s}$ )

The suction region created by the lower vortex structure can be seen below $\mathrm{h} / \mathrm{H}=0.4$ in Figure 8 . The flow is largely two dimensional across the width of the model, with the presence of some three dimensional structures in the lower corners of the base. Figure 8 shows that when 4 slats are added the surface pressures become much more uniform across the width of the model, especially in the upper half of the base. It also seems to show a global increase in $\mathrm{C}_{\mathrm{p}}$, with the majority occurring within the upper base region. In order to more easily identify where increases in pressure occur the baseline pressure distribution has been subtracted from the pressure distribution for 3 slats, and the distribution for 4 slats, and the resulting plots are shown in Figure 9 and Figure 10 respectively.

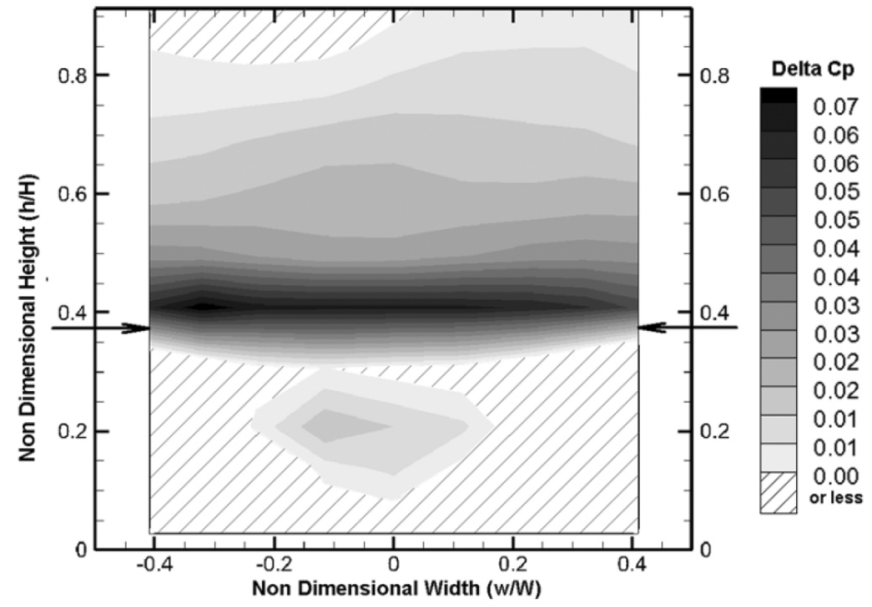

Figure 9. Change in model base pressure relative to baseline configuration with 3 slats fitted (freestream velocity $40 \mathrm{~m} / \mathrm{s}$ )

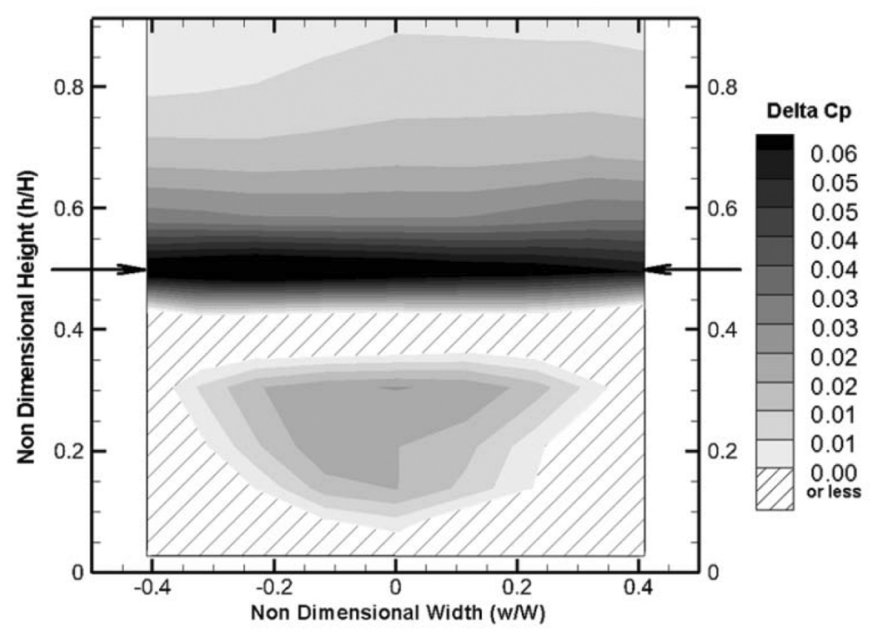

Figure 10. Change in model base pressure relative to baseline configuration with 4 slats fitted (freestream velocity $40 \mathrm{~m} / \mathrm{s}$ )

For clarity any areas that showed no change or a slight decrease in pressure are identified by a hatched region, and the position of the uppermost slat is identified in each plot by two arrows. It is immediately apparent that a large region of increased pressure occurs along the width of the upper slats. It appears that the reversed flow from the lower vortex seen in Figure 1 and Figure 2 impinges on the upper slat when it recirculates back around towards the base surface. In doing so it creates a region of increased pressure above the slat which spreads upwards on the upper half of the base. Note that the plots do not show an exact distinction between the pressure above and below the upper slat because of interpolation between tapping locations applied when generating the contours in Figure 9 and Figure 10. Additionally the suction at the centre of the lower vortex region between $\mathrm{x} \approx-0.2$ to 0.2 
and $\mathrm{y} \approx 0.1$ to 0.5 in Figure 10 shows an increase in pressure. This pressure increase may be associated with a reduction in the rotational energy of the structure, and hence the velocity across the base surface.

\section{FULL SCALE RESULTS}

While it was not the intention to directly reproduce the model scale tests at full scale in order to replicate the body model surfaces as closely as possible the full scale vehicle was fitted with a flat floor. This was considered important in order to generate stable recirculating structures within the wake of the vehicle. It is worthy of note that the addition of the flat floor alone created a drag reduction of 32 counts, which adds weight to the argument for flats floors being fitted on production vehicles. This magnitude of drag reduction also supports the findings of previous work $(\underline{12})$.

When slats were added to the base of the vehicle without a smooth underfloor in place there was no change in drag that could be considered to be outside of measurement error $( \pm 1.5$ counts). This result illustrates that the mechanisms targeted by the addition of small slats in these tests are highly dependent on the conditioning of the flow before it separates from the rear edges of the vehicle. It suggests that if the separation location/line all the way around the vehicle is transient rather than fixed, the wake structures targeted never become sufficiently stable for them to be influenced by the addition of the slats.

Figure 11 shows the effect of adding up to six slats (configuration 1) and removing them at each configuration from the top down, when the vehicle was fitted with a flat floor. When six slats are in place (configuration 1) there is a drag reduction of up to 4 counts, however when the upper slat is removed in configurations 2-6 there is no reduction in drag. These results indicate that in order to achieve a change in drag it is either the upper slat that is important or the presence of all the slats that cause the change.

Figure 12 shows the changes in lift when slats are added and although lift is reduced when all six slats are in place by up to 10 counts, it is also the case that lift is reduced in configurations 2-6 where no changes in drag were observed. It is clear some modification to the flow structure around the rear of the vehicle and in the wake must be occurring to create the changes in drag seen in configuration 1 and the consistent changes in lift seen throughout configuration changes 2-6.

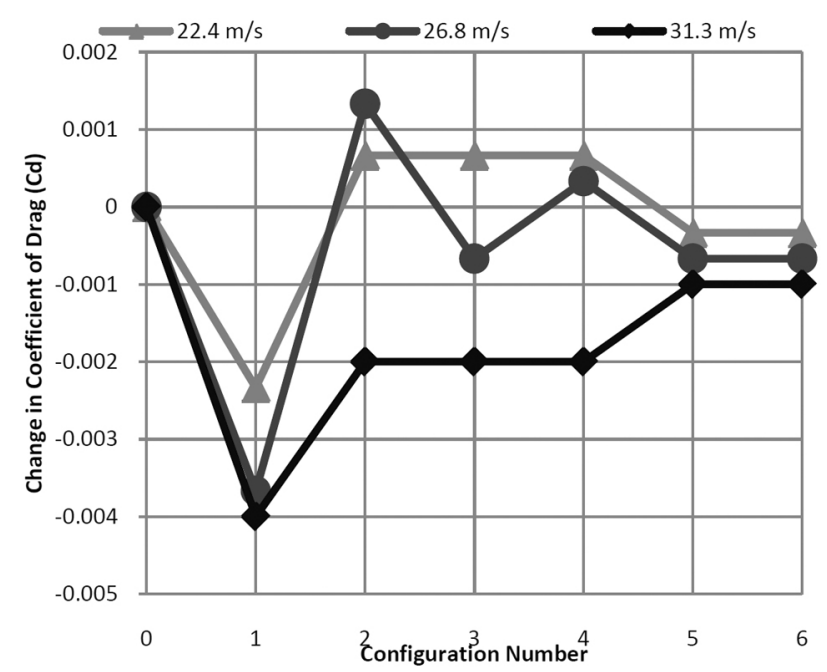

Figure 11. Change in Cd vs configuration number

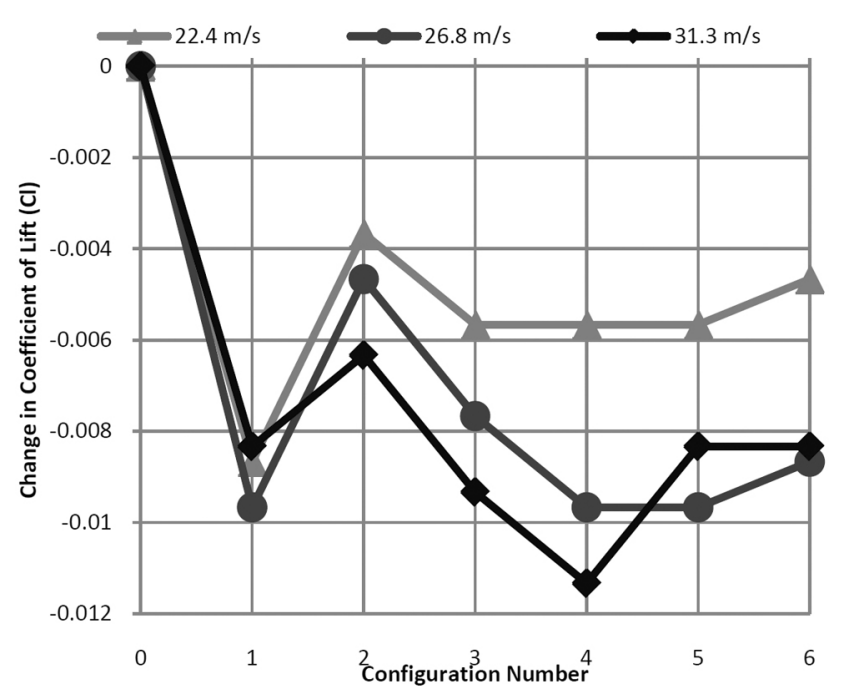

Figure 12. Change in $\mathrm{Cl}$ vs configuration number

Figure 13 shows the changes in base pressure for configuration 1 relative to the base pressures of the baseline configuration. A global base pressure increase occurs above $0.2 \mathrm{H} / \mathrm{h}$ from the floor of the vehicle, whilst an increase in suction occurs across the lower $20 \%$ of the base region. The changes do not show the high concentration of high pressure across the width of a single slat as seen at model scale in Figure 9 and Figure 10, indicating that the means by which the base pressures are modified may be different. However, the slats applied are a uniform two dimensional physical addition across the width of the base, and the resulting changes in base pressure are relatively uniform across the width of the vehicle. 


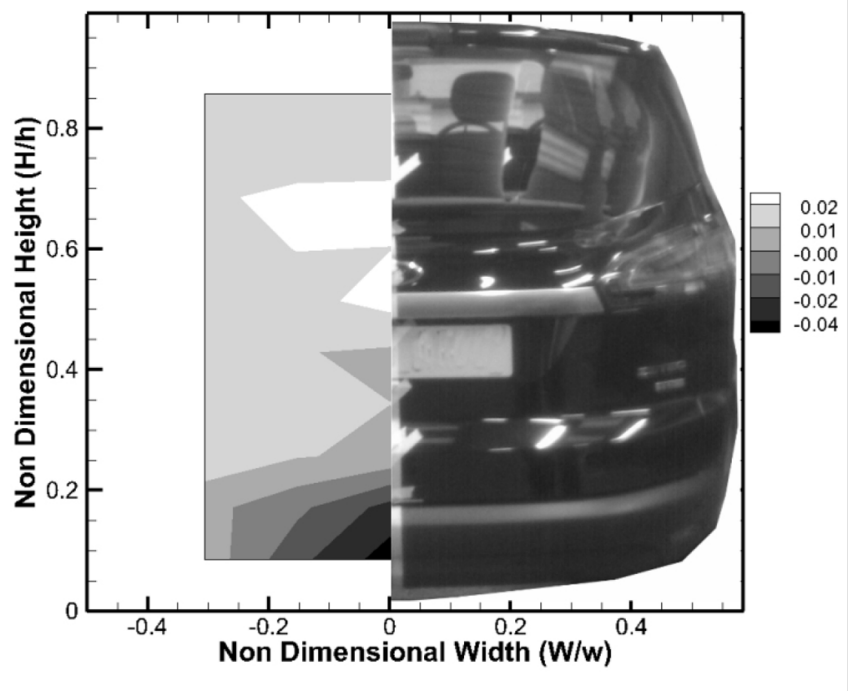

Figure 13. Change in base pressure (Cp) with 6 slats applied

Examination of the baseline base pressure distribution individually infers a three dimensionality of the wake flow. When 6 slats are added the individual base pressure distribution for configuration 6 shows a much more uniform wake flow structure across the width of the base.

\section{DISCUSSION}

The changes implemented in the current test are physically small and located in the wake of a squareback vehicle. Such small modifications in the wake region would not normally be addressed in an aerodynamic optimisation process since small changes on the base region of a vehicle have previously be considered unimportant. Any changes in this region which have previously been effective have required large physical changes, or trailing edge modifications such as tapering or tripping. With this in mind the reductions in lift and drag as a result of the configurations tested have been surprisingly large.

The pressure results in Figure 9 and Figure 10 and the PIV images of the flow structure in Figure 1 and Figure 2 go some way to explaining how the wake structures can interact with the base slats to cause body force changes in the scale model tests. However an area weighted calculation based on the contribution from these small slats shows that the changes in lift cannot be explained by pressure changes on the horizontal surfaces of the slats themselves, due to their small horizontal area (as a percentage of the whole vehicle horizontal area). Further investigation using tappings located on the floor and roof of the model may identify if the lift reduction using the base slats is caused by pressure changes on the floor of the model by accelerating flow underneath it.
The mechanisms involved in creating the changes on the full scale model are still not understood, however it is clear that small changes in the physical architecture of the vehicle/ model relationship can have an effect on where the base slats are effective. This is thought to be due to the differences in the wake structures found in the two experiments. None of the base slats tested at full scale without a flat floor had an effect, again highlighting the sensitivity to wake structure changes.

The use of moving ground simulation and rotating wheels will also influence the flow under the vehicle and the structure of the wake. As a result the optimum position of the slats may be further influenced by these changes in the test conditions, which may have to be addressed in any vehicle optimization process.

From the tests described in Figure 2 and the work carried out here, it is clear that wake size manipulation is not the only means of creating base pressure recovery and lift changes, and detailed optimisation on the base surface may be a means of further aerodynamic optimisation. The flow mechanisms involved in creating the observed changes are still not fully understood and the transient nature of wake flows makes the understanding a difficult task. However it is clear that with an optimisation process applied to a specific vehicle shape, drag and lift modification may be possible.

\section{CONCLUSIONS}

A small passive modification has been tested in $1 / 4$ scale and full scale wind tunnel tests. The addition of low profile horizontal slats to the base surface of the scale model produced drag reductions of up to 8 counts and lift reductions of up to 32 counts.

Pressure measurements on the base of the model showed that the interaction of the upper slat with the recirculating vortex structure in the wake creates a region of increased pressure above the upper slat. Additionally pressure was recovered in the lower half of the base where the suction regions associated with the presence of the lower vortex structure are located.

At full scale the addition of horizontal slats to the base of the vehicle created drag reductions of 4 counts and lift reductions of 10 counts. Tests conducted without a smooth underfloor showed little or no change to the lift and drag forces.

In the full scale configurations where force changes were observed pressure measurements showed a global recovery of base pressure on the base above $0.2 \mathrm{H} / \mathrm{h}$ from the floor of the vehicle; below $\mathrm{h} / \mathrm{H}=0.2$ a reduction in base pressure was observed. A lack of understanding of the standard full scale vehicle wake structure means the aerodynamic force changes 
observed cannot be explained as easily as the scale model results.

Further investigation is required to fully understand how such a small passive modification can cause such large force changes observed at full scale.

\section{REFERENCES}

1. Golke, M. "Drag reduction by flow control at PSA Peugeot-Citroen" KATnetII Separation and Control Workshop, Toulouse, France 2008

2. Schultz, S. "Aerodynamics of Modern Sport Utility Vehicles" $8^{\text {th }}$ MIRA International Vehicle Aerodynamics Conference 2010

3. Hucho, W.-H. "Aerodynamics of Road Vehicles," SAE International, Warrendale, PA, ISBN 978-0-7680-0029-0, 1998.

4. Ribaldone, E. et al, "Application of mulit-objective optimization to the aerodynamic development of passenger vehicles at FIAT" $8^{\text {th }}$ MIRA International Vehicle Aerodynamics Conference 2010

5. Schrefl, M. "The Audi A1 - Aerodynamics and Aeroacoustics" MIRA International Vehicle Aerodynamics Conference 2010

6. Littlewood, R.P., Passmore, M. A. "Aerodynamic drag reduction of a simplified squareback vehicle using steady blowing" Submission for Journal of Experiments in Fluids 2011

7. Littlewood, R. and Passmore, M., "The Optimization of Roof Trailing Edge Geometry of a Simple Square-Back.," SAE Technical Paper 2010-01-0510, 2010, doi: 10.4271/2010-01-0510.

8. Roshko, A. "Perspective on Bluff Body Aerodynamics" Journal of Wind Engineering and Industrial Aerodynamics, 49 (1993) 79-100

9. Roumeas, A, Gillieron, Azeddine, K. "Analyse and Control of the Near-Wake Flow over a Square Back Geometry" $3^{\text {rd }}$ AIAA Flow Control Conference 5-8 June 2006, San Francisco, California, AIAA 2006-3336

10. Le Good, G. and Garry, K., "On the Use of Reference Models in Automotive Aerodynamics," SAE Technical Paper 2004-01-1308, 2004, doi:10.4271/2004-01-1308.

11. Duell, E. and George, A., "Measurements in the Unsteady Near Wakes of Ground Vehicle Bodies," SAE Technical Paper 930298, 1993, doi: 10.4271/930298.

12. Le Good, G., Howell, J., Passmore, M., and Garry, K., "On-Road Aerodynamic Drag Measurements Compared with Wind Tunnel Data," SAE Technical Paper 950627, 1995, doi: $10.4271 / 950627$.

\section{CONTACT INFORMATION}

Rob Littlewood

R.P.Littlewood@lboro.ac.uk

Martin Passmore

M.A.Passmore@1boro.ac.uk

Dan Wood

D.W.Wood@lboro.ac.uk

\section{ACKNOWLEDGMENTS}

The authors would like to thank Rob Hunter and Stacey Prentice for their help with production of the wind tunnel model, Adrian Gaylard and Steve Windsor for their time and interest in the configurations tested. They would also like to thank Dave, Ian and Ivan for their assistance with the full scale wind tunnel testing, and Jeff Howell for his continued guidance.

\section{DEFINITIONS/ABBREVIATIONS}

$\mathbf{c}_{\mathbf{d}}$

Coefficient of drag

$\mathbf{c}_{1}$

Coefficient of lift

\section{Count}

$1 \times 10^{-3} \mathrm{C}_{\mathrm{d}}$ or $\mathrm{C}_{1}$

H

Model or vehicle body height (m)

$\mathbf{h} / \mathbf{H}$

Non dimensional vehicle or body height

W

Model or vehicle body width (m)

$\mathbf{w} / \mathbf{W}$

Non dimensional vehicle or body width

PIV

Particle Image Velocimetry

NACA0021

Symmetric wing profile with $21 \%$ thickness to chord length ratio 
The Engineering Meetings Board has approved this paper for publication. It has successfully completed SAE's peer review process under the supervision of the session organizer. This process requires a minimum of three (3) reviews by industry experts.

All rights reserved. No part of this publication may be reproduced, stored in a retrieval system, or transmitted, in any form or by any means, electronic, mechanical, photocopying, recording, or otherwise, without the prior written permission of SAE.

ISSN 0148-7191
Positions and opinions advanced in this paper are those of the author(s) and not necessarily those of SAE. The author is solely responsible for the content of the paper.

SAE Customer Service:

Tel: 877-606-7323 (inside USA and Canada)

Tel: 724-776-4970 (outside USA)

Fax: 724-776-0790

Email: CustomerService@sae.org

SAE Web Address: http://www.sae.org

Printed in USA 